\title{
Cetuximab/Irinotecan/Vemurafenib Regimen
}

National Cancer Institute

\section{Source}

National Cancer Institute. Cetuximab/Irinotecan/Vemurafenib Regimen. NCI Thesaurus.

Code C153480.

A chemoimmunotherapy regimen consisting of cetuximab, irinotecan and vemurafenib that can be used for the treatment of colon cancer. 